\title{
The Right Isotherms for the Right Reasons? Validation of Generic Force Fields for Prediction of Methane Adsorption in Metal-Organic Frameworks
}

\author{
Matthew J. Lennox ${ }^{a}$, Michelle Bound ${ }^{b}$, Alice Henley ${ }^{b}$ and Elena Besley ${ }^{b *}$ \\ ${ }^{a}$ Centre for Advanced Separations Engineering, Department of Chemical Engineering, University of \\ Bath, Bath, BA2 7AY, UK \\ ${ }^{b}$ Computational and Theoretical Chemistry, School of Chemistry, University of Nottingham, \\ Nottingham, NG7 2RD, UK
}

\begin{abstract}
In recent years, the use of computational tools to aid in the evaluation, understanding and design of advanced porous materials for gas storage and separation processes has become ever-more widespread. High-performance computing facilities have become more powerful and more accessible and molecular simulation of gas adsorption has become routine, often involving the use of a number of default and commonly-used parameters as a result. In this work, we consider the application of molecular simulation in one particular field of adsorption - the prediction of methane adsorption in metal-organic frameworks in the low-loading regime - and employ a range of computational techniques to evaluate the appropriateness of many commonly chosen simulation parameters to these systems. In addition to confirming the power of relatively simple generic force fields to quickly and accurately predict methane adsorption isotherms in a range of MOFs, we demonstrate that these force fields are capable of providing detailed molecular-level information which is in very good agreement with quantum chemical predictions. We highlight a number of chemical systems in which molecular-level insight from generic force fields should be approached with a degree of caution and provide some general recommendations for best-practice in simulations of $\mathrm{CH}_{4}$ adsorption in MOFs.
\end{abstract}

Keywords: methane adsorption, binding, DFT, force field validation, metal-organic frameworks Introduction 
Over the past several decades, the design, synthesis and development of new metal-organic frameworks (MOFs) has garnered a great deal of attention in scientific literature. Their flexible 'mixand-match' construction - based on a combination of one or more types of metal node coordinated with one or more types of organic ligand - allows for a wide range of topologies, pore sizes, surface areas and chemical environments, as well as an ever-increasing variety of applications, including catalysis, drug delivery, gas storage and separations $[1,2,3,4,5,6]$. The huge number of potential MOF structures, combined with the range of complex chemical, material and mechanical phenomena observed therein has resulted in computational tools playing an increasingly important role in MOF science $[7,8,9,10,11]$. In the present work, we focus on one particular application: the computational prediction of $\mathrm{CH}_{4}$ adsorption in the low loading regime in MOFs.

In 2004, the Snurr group $[12,13]$, employed molecular simulations to evaluate the adsorption of methane in a range of real and hypothetical MOF materials and demonstrated that, in the case of IRMOF-1 and IRMOF-6, grand canonical Monte Carlo (GCMC) simulations were able to reproduce the experimental methane adsorption isotherms to within 5-10\%. Since then, GCMC has been employed to predict $\mathrm{CH}_{4}$ adsorption isotherms in a wide range of MOFs and related structures $[14,15,16,17,18$, $19,20,21,22,23]$, with reasonable agreement with experiment reported in many cases.

One of the oft-quoted beauties of molecular simulation is that, when a simulated isotherm which is in reasonable agreement with experiment is recovered, it is possible to extract accurate and physically meaningful information regarding the preferred adsorption sites and energetics of the adsorption process from these simulations - information which is typically extremely challenging or impossible to obtain from experimental studies. The tacit assumption being that recovering the correct isotherm means that the underlying chemical/mathematical description is correct - that is, one can only correctly predict the isotherm if the descriptions of the atomic interactions and strengths are also correct. In the present work, we attempt to shed light on this fundamental assumption in the case of $\mathrm{CH}_{4}$ adsorption in a range of MOFs following a multi-level computational approach. The suitability of three common generic force fields (UFF[24], DREIDING[25] and OPLS-AA[26, 27]) for the prediction of macroscopic properties (adsorption isotherms) is evaluated against available experimental data, while the recovery of accurate atomistic-level information from force field calculations (small molecule interactions) is compared to density functional theory (DFT) calculations. While all three force fields perform reasonably well in both aspects of the study, we highlight a number of systems in which these generic force fields should be approached with caution before, finally, making some general recommendations for good practice in the choice of generic force field as applied to methane adsorption in MOFs.

\section{Simulation Details}

The present work is not intended to provide a comprehensive review of simulations of adsorption in MOFs - several excellent review articles discuss this subject $[8,10,28]$ and the reader is directed towards these for more detail. It is necessary, however, to briefly introduce some of the technical aspects of simulations of $\mathrm{CH}_{4}$ adsorption in MOFs. In general, two broad classes of simulation are available: approaches in which the interactions between atoms are described using quantum chemical or ab initio derivations, and those using some combination of empirically derived force fields (so-called 'classical' approaches). Due to the relatively high computational cost - and thus small system sizes - associated with quantum chemical methods, adsorption properties are typically assessed using classical molecular simulations. 
The adsorption of $\mathrm{CH}_{4}$ in a range of MOF-based systems (see $\mathrm{SI}$ ) was evaluated in three different simulation environments. The interaction of single gas molecules with fragments of the MOF material was evaluated using DFT. The same single gas molecule - ligand interactions were also probed using analogous force-field (FF) based approaches, in which the dependence of gas-MOF interaction on location and guest orientation was studied. Finally, the adsorption isotherm was evaluated in the periodic MOF system using grand GCMC simulations and, where possible, compared to experimental adsorption data.

\section{Density Functional Theory Calculations}

The interaction of a single $\mathrm{CH}_{4}$ molecule with fragments of the MOF was investigated using DFT with Grimme 3 dispersion correction[29] implemented in the Q-Chem software package[30]. In most cases, the fragment was the aromatic core of the ligand used in the MOF, with carboxylate groups replaced with either methyl groups or hydrogen. In order to fully investigate the interaction of guest molecules near the oxygen atoms of the carboxylate groups, several calculations were undertaken in which the fragment was the $\mathrm{Zn}$-benzoate cluster typical of IRMOF-1. The interaction of the guest molecule with the fragment was evaluated in two steps, both using the B3LYP functional[31], which has been shown to be suitable for the treatment of weakly bound light gas-aromatic systems[32,33] as well as the interaction between $\mathrm{CH}_{4}$ and unsaturated metal centres in MOFs[34, 35]. Geometry optimization of the guest-linker dimer was undertaken using the $6-31+G^{*}$ basis set, followed by single-point energy calculations using the larger $6-311+G *$ basis set, from which the binding energy of the guest molecule was estimated following the counterpoise method for the correction of the basis set superposition error[36]. For each system, several initial geometries were evaluated and in all calculations the atoms of the linker fragment were kept fixed, while the guest molecule and its constituent atoms were allowed to adjust position upon optimisation. Thus, for each system, a range of binding locations were investigated and the strongest binding locations identified.

\section{Force Field-Based Calculations}

At the core of any classical molecular simulation is the choice of mathematical functions used to describe atomic interactions. In the case of the relatively simple and non-polar methane molecule, the 12-6 Lennard-Jones (L) form (1) is the most common choice.

$$
v_{i j}\left(r_{i j}\right)=4 \varepsilon_{i j}\left[\left(\frac{\sigma_{i j}}{r_{i j}}\right)^{12}-\left(\frac{\sigma_{i j}}{r_{i j}}\right)^{6}\right]
$$

Where the total energy of interaction $\left(u_{\mathrm{ij}}\right)$ between atoms $i$ and $j$ is a function of their separation distance $\left(r_{i j}\right)$. The minimum of the potential (which primarily governs the strength of interaction) and the distance at which $v_{\mathrm{ij}}=0$ (which is conceptually related to the size of the atoms) are donated by $\varepsilon_{\mathrm{ij}}$ and $\sigma_{\mathrm{ij}}$ respectively. The cross-terms, $\varepsilon_{\mathrm{ij}}$ and $\sigma_{\mathrm{ij}}$, of equation (1) are typically calculated by combining $L$ parameters for the individual species $i$ and $j$ following some well-defined mixing rule. The total interaction energy of the system is considered to be a pair-wise summation of all atom pairs.

In simulations of adsorption, the choice of $\varepsilon_{\mathrm{ij}}$ and $\sigma_{\mathrm{ij}}$ determines the strength of interaction both between sorbate and adsorbent and between sorbate molecules, as well as the volume of 
individual molecules and the pore volume available for adsorption. Several sets of $L$ parameters for $\mathrm{CH}_{4}$ have been derived which are capable of predicting the behaviour of the bulk fluid in simulation, of which the TraPPE force field[37] is most commonly applied in studies of adsorption. Although a number of groups have derived framework $L$ parameters for specific MOF-guest systems[38, 39, 40], the majority of studies make use of one of several generic force fields, of which the most common are UFF, DREIDING and OPLS-AA. Both UFF and DREIDING were developed and tested for their ability to predict crystal structures, bond lengths and bond angles for organic[25, 41] and, in the case of UFF, organometallic molecular complexes[42], while OPLS-AA was developed to correctly reproduce properties of bulk organic liquids, such as the heat of vaporisation and liquid density[26, 27]. It should be noted that while all three force fields have been used to simulate gas adsorption in MOFs with some degree of success, none of them were designed to describe the interaction between a relatively isolated organic fragment or metal cluster with adsorbed species and, therefore, should not be assumed to be transferable to all MOF systems.

Unless stated otherwise, force-field based calculations in this work were undertaken using the TraPPE parameters for $\mathrm{CH}_{4}$, which was treated using a united atom [UA] description[37]. In the case of UA methane, only a $U$ component was considered. Select systems (explicitly identified in later sections) were further evaluated using the $\amalg$ parameters of the OPLS-AA $\mathrm{CH}_{4}$ model[27], which incorporates both $\mathrm{L}$ and electrostatic components. In the evaluation of gas-ligand binding using classical methods, the CHELPG partial charges derived from the complementary DFT calculations were used to describe the MOF fragment. Three primary sources of $L$ parameters for the organic portion of the framework were explored: UFF, DREIDING and OPLS-AA. As is typical in the MOF literature, metal atoms were described using UFF parameters, as these parameters are often not available in the DREIDING or OPLS force fields. All LJ parameters used in this work are listed in SI.

While the primary variable which we adjust in the present work is the choice of $\mathrm{LJ}$ parameters for framework atoms, in truth we are evaluating the ability of the combined MOF-guest LJ terms following Lorentz-Berthelot mixing rules[43] - to describe the MOF-gas interaction. The TraPPE force field was developed to describe the bulk properties of the adsorbed gases, and has been shown to capture the adsorption isotherm well in the medium to high loading regimes in a wide range of MOFs[18, 44, 45]. We thus consider it is most sensible to assign the prediction of the low loading regime and guest-framework interactions to the choice of framework $L$ parameters, though this is not the only approach which one may take.

For each of the systems evaluated via DFT, complementary calculations were undertaken using classical, FF-based methods implemented in an in-house modified version of the Kh_d toolset[46]. In these simulations, the optimised MOF fragment from the DFT simulation was placed in a large simulation box. The box was then discretised on a $0.2 \AA$ grid and, for each point on the grid, the interaction of the guest molecule with the fragment was evaluated using the chosen force field. In the case of single-atom molecules (ie united atom $\mathrm{CH}_{4}$ ), only a single calculation was performed per point. For polyatomic species, $\mathbf{5 0 0 0}$ randomly generated trial orientations were tested per point and both the orientational averaged interaction energy and the individual configurations resulting in the strongest interaction were recorded. Both the fragment and probe molecule were treated as rigid bodies.

\section{Grand Canonical Monte Carlo}


For those MOFs for which experimental adsorption data was available, theoretical adsorption isotherms for $\mathrm{CH}_{4}$ were generated via GCMC simulations carried out using the MuSiC software package[47]. Each simulation point was allowed at least $6 \times 10^{6}$ Monte Carlo steps to come to equilibrium and system properties were evaluated over a further $10 \times 10^{6}$ steps. Input fugacities were calculated using the Peng-Robinson equation of state and framework atoms were kept fixed at their crystallographic positions (i.e. the MOF was assumed to be rigid).

\section{Results and Discussion}

\section{Prediction of Adsorption Isotherms}

Of the fourteen ligands included in the DFT/FF comparative study, several belong to MOF structures for which reliable low pressure $\mathrm{CH}_{4}$ adsorption data at $273 \mathrm{~K}$ is available (IRMOF-8, MFM-188, MFM-181, MFM-183, MFM-185 and UTSA-33). Of these, a similar force field evaluation has been previously undertaken for IRMOF-8[48]. For each MOF, methane adsorption isotherms were simulated using UFF, OPLS-AA and DREIDING force fields (see SI). In subsequent analysis, we report adsorbed amounts as the absolute number of methane molecules per unit cell for both experimental and simulated data. In order to compare force field performance across different MOF systems, we report the deviation of simulation from a Langmuir isotherm fitted to experimental data. In all cases the $R^{2}$ value of the fitted isotherm was $>0.97$. Fractional loading, $\vartheta$, is defined with respect to the fitted saturation capacity of the experimental system.

The isotherms recovered for MFM-181 (Fig. 1) are representative of the trends observed in all seven systems. As has been noted previously in the case of covalent organic frameworks[20] and IRMOF-8[48], UFF tends to significantly over-estimate adsorbed amounts compared to DREIDING, by between 15 and $50 \%$. The OPLS-AA force field performs very similarly to UFF, over-estimating compared to DREIDING by between $8 \%$ and $40 \%$. The largest discrepancy is evident at low fractional loadings $(\vartheta<0.25)$ and in all cases, the three force fields move towards convergence at higher pressures as the adsorption process begins to be dominated by methane-methane rather than methane-framework interactions. 


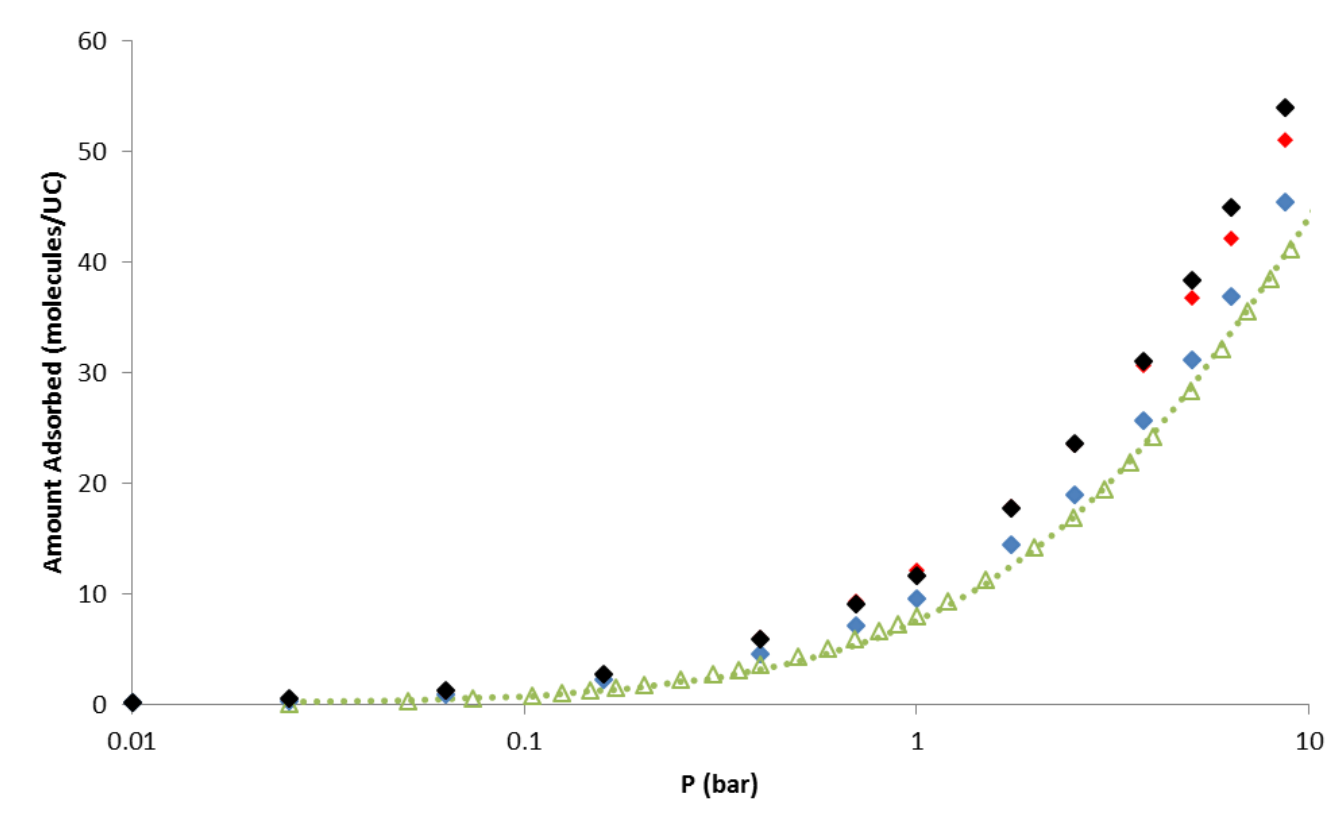

Figure 1 - Experimental[49] (green triangles) and simulated (DREIDING - blue; OPLS - red; UFF black) $\mathrm{CH}_{4}$ adsorption isotherms at $273 \mathrm{~K}$ in MFM-181. The Langmuir fit of the experimental data is indicated by the green dashed line.

In all cases, the isotherms predicted from generic force fields were in qualitative agreement with experimental data in the low and medium loading regimes, here taken as fractional loadings $\theta<$ 0.5. DREIDING was found to provide the closest quantitative agreement with experiment (Fig. 2), though still over-estimates adsorbed amounts compared to experiment by $15-36 \%(\vartheta<0.25)$ and 13 $14 \%(0.25 \leq \vartheta<0.5)$. It is worth restating that these percentages relate to the absolute number of molecules present in the unit cell. For the low loading levels and MOFs considered in this study, the difference between experiment and simulation is in the region of 5-10 molecules per unit cell (2-3 $w t \%$, or $10-40 \mathrm{~cm}^{3}$ sтp $/ g$, although these values are, of course, strongly dependent on the density of the MOF system.

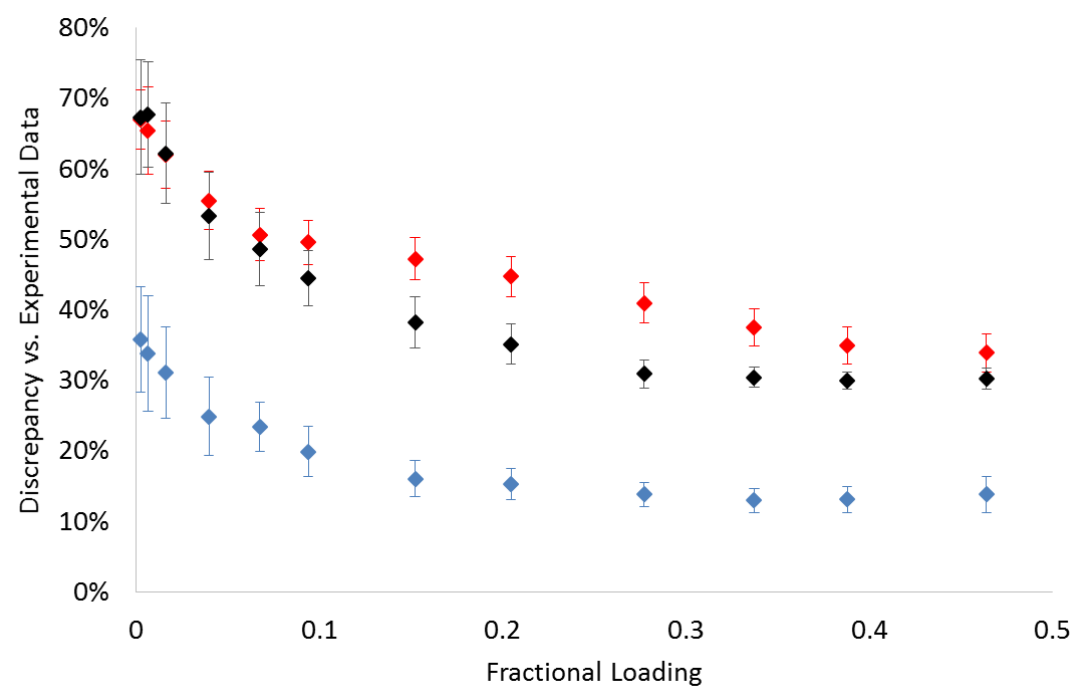


Figure 2 - Average relative deviation between experimental and predicted adsorption isotherms as a function of fractional loading. Blue - DREIDING; red - OPLS; Black - UFF.

OPLS and UFF performed very similarly with respect to quantitative agreement with experiment (Fig. 2), over-estimating by an average of $43 \%$ (OPLS) and $38 \%$ (UFF) for $\vartheta<0.25$, and $27 \%$ (OPLS) and $24 \%$ (UFF) for $0.25 \leq \vartheta<0.5$. Both force fields have been previously reported to overpredict the adsorption of methane and other gases with respect to experiment in a range of other systems. Yang and Zhong[50] reported an over-estimation in the cases of $\mathrm{CH}_{4}$ on IRMOF-1 and Cu-BTC using OPLS-AA and suggested a re-parameterisation of the $\mathrm{C}$ and $\mathrm{O}$ atoms of the carboxylate groups, reducing $\varepsilon_{i i}$ by as much as $30 \%$. A similar reduction in $\varepsilon_{i i}$ across all framework atoms was suggested for UFF by Fairen-Jimenez et $a /[51]$ and Pérez-Peritello et al[23] (who scaled $\varepsilon_{\text {UFF }}$ by 0.59 and 0.69 respectively) based on simulations of methane adsorption in ZIF-8 and ZIF-69, while a similarly large over-estimation ( $\sim 50 \%$ ) has been reported in UiO-66(Zr) using UFF[22]. We would suggest that while UFF and OPLS-AA are suitable for qualitative prediction of adsorption isotherms in MOFs, both are likely to over-estimate the amount adsorbed, as well as low-coverage properties such as heats of adsorption or Henry's constants, by a significant amount.

Although DREIDING has been shown to significantly over-estimate methane adsorption when compared to experiment in ZIF-8[23], very good agreement was observed in the case of UiO-66(Zr)[22] $(\sim 9-20 \%$ over-estimation for $\vartheta<0.5)$ and in the present work, while simulations in IRMOF-1 and IRMOF-6[12] and in IRMOF-8[48] found excellent quantitative agreement with experiment $(5-10 \%$ difference when averaged over the full isotherm). While there are too many variables to fully rationalise these apparent differences in quantitative agreement (e.g. the quality of the experimental data and experimental sample, the physical characteristics and composition of the MOF and the choice of simulation software), we are able to investigate the influence of one particular simulation parameter on the computed isotherms, namely the choice of cut-off radius, i.e. the atomic separation beyond which $\mathrm{L}$ interactions are assumed to be negligible.

The cut-off radius $\left(r_{\mathrm{c}}\right)$ is typically expressed in terms of multiples of the largest $L \sigma$ parameter used in the simulation $-\sigma$ for methane in this work. Suggested values of $r_{\mathrm{c}}$ range from $2.5 \sigma$ for typical $\checkmark$ fluids, which can be expected to introduce an error in the total energy of the system in the region of $10 \%[52]$, to $5.5 \sigma$ in $M C$ simulations of vapour-liquid coexistence[53, 54]. In the present work, $r_{\mathrm{c}}=15 \AA(4 \sigma)$ was implemented, while in the systems described above, the cut-off radius ranged from 12.8 to $18 \AA$ ( $3.4 \sigma$ to $4.8 \sigma)$. Following the work of Düren et al[12], $12.8 \AA$ has proven a popular choice of cut-off radius in simulations of gas adsorption in MOFs. It is worth noting that this radius corresponds to just under half the width of the unit cell of IRMOF-1 and thus represented a compromise between computational accuracy and efficiency. Further increase in the cut-off radius would have required a significantly larger simulation box (eight unit cells instead of one) and increased CPU time as a result. In order to better understand the influence of cut-off radius on adsorbed amounts, methane adsorption isotherms were simulated in the systems previously introduced, as well as IRMOF-1, using the DREIDING force field and a cut-off radius ranging from 10 to $25 \AA$ ( $2.6 \sigma$ to $6.7 \sigma$ ).

For all systems studied, increasing the cut-off radius was found to increase the amount adsorbed across the full pressure range considered. The recovered isotherms begin to converge, both in terms of the number of adsorbed molecules and total system energy, for a cut-off radius of $15 \AA$ and are statistically indistinguishable for cut-off radii of $20 \AA$ and above (Fig. 3). For a cut-off radius of 
$15 \AA$, the simulated isotherms were quantitatively accurate to within $5 \%$ of those recovered using a $25 \AA$ A cut-off. This accuracy improves to within $3.5 \%$ for a cut-off of $17 \AA$.

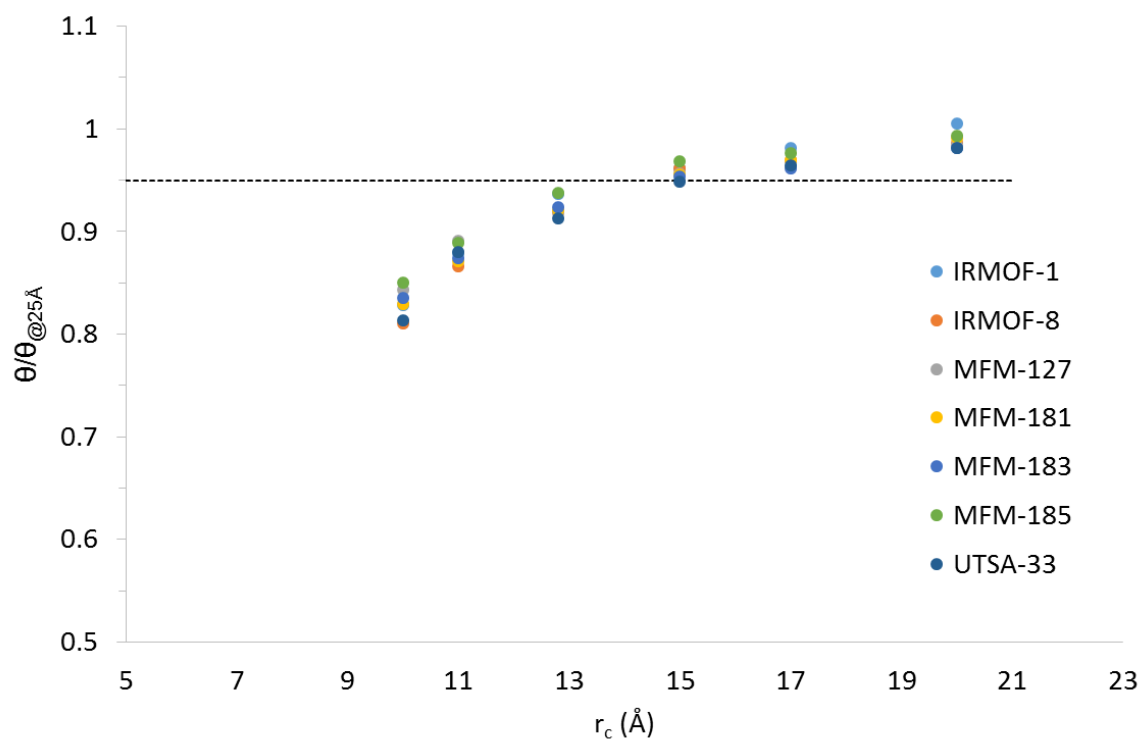

Figure 3 - Dependence of amount adsorbed on cut-off radius, averaged for each system over the entire pressure range $(0-70 \mathrm{bar})$. The ratio of the fractional loading predicted at a given cut-off, $r_{c}$, to that predicted in the same system using a cut-off of $25 \AA$, is shown on the $y$-axis. The dashed line indicates a ratio of 0.95 .

As illustrated for IRMOF-1 (Fig. 4), the recovered isotherms in each MOF are all qualitatively similar and qualitatively correct in comparison to the experimental isotherm, even for the smallest cut-off implemented $\left(r_{c}=10 \AA\right)$. For the smallest cut-off used, the excess amount adsorbed is 10$30 \mathrm{~cm}^{3}$ Sтр/g lower than that predicted in simulations using larger cut-off radii $\left(r_{c}>15 \AA\right)$. Furthermore, the adsorption mechanism and predicted adsorption sites are identical in all cases.

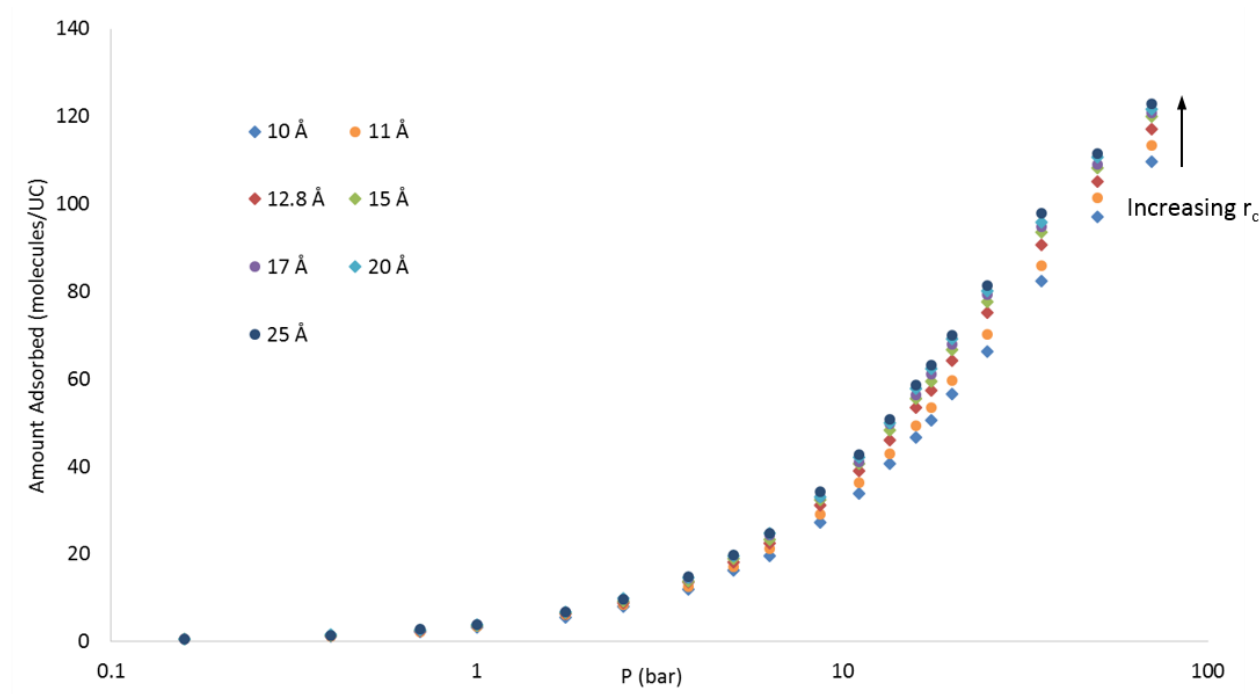

Figure 4 - Simulated $\mathrm{CH}_{4}$ isotherms in IRMOF-1 at $273 \mathrm{~K}$ for $r_{\mathrm{c}}$ ranging from 10 to $25 \AA$ 
We suggest that while a $12.8 \AA$ cut-off is likely to introduce a statistically significant underestimation of the amount adsorbed, the isotherm is likely to fall within $10 \%$ of the converged adsorption isotherm and in no way invalidates predictions of capacity or suitability for methane adsorption applications. We would recommend, however, that a cut-off of at least $15 \AA(4 \sigma)$, and preferably greater than $17 \AA(4.5 \sigma)$ be implemented in future work. The influence of cut-off radius does exhibit some system-dependence, however, and care should be taken to ensure these values are appropriate for the system of study.

Although all three force fields thus appear to over-estimate the strength of interaction between methane and the framework, they typically predict isotherms which are in reasonable agreement with experiment. We now consider whether having an isotherm which looks correct means that the associated atomistic detail of the prediction is physically insightful and chemically accurate for these systems at low loading (i.e. relatively isolated methane molecules interacting with the MOF). When evaluating the accuracy of these atomistic-level predictions, it is helpful to consider two classes of system - those whose ligand cores are based purely on carbon and hydrogen, and those whose cores incorporate other elements (oxygen, sulphur and nitrogen in this study). Note that the ligand fragments evaluated here have been methyl-, rather than $\mathrm{COOH}$-terminated. The influence of the omitted carboxylate group - which is of particular relevance to the OPLS-AA force field - is evaluated subsequently in the case of the Zn-benzoate cluster typical of IRMOF-1.

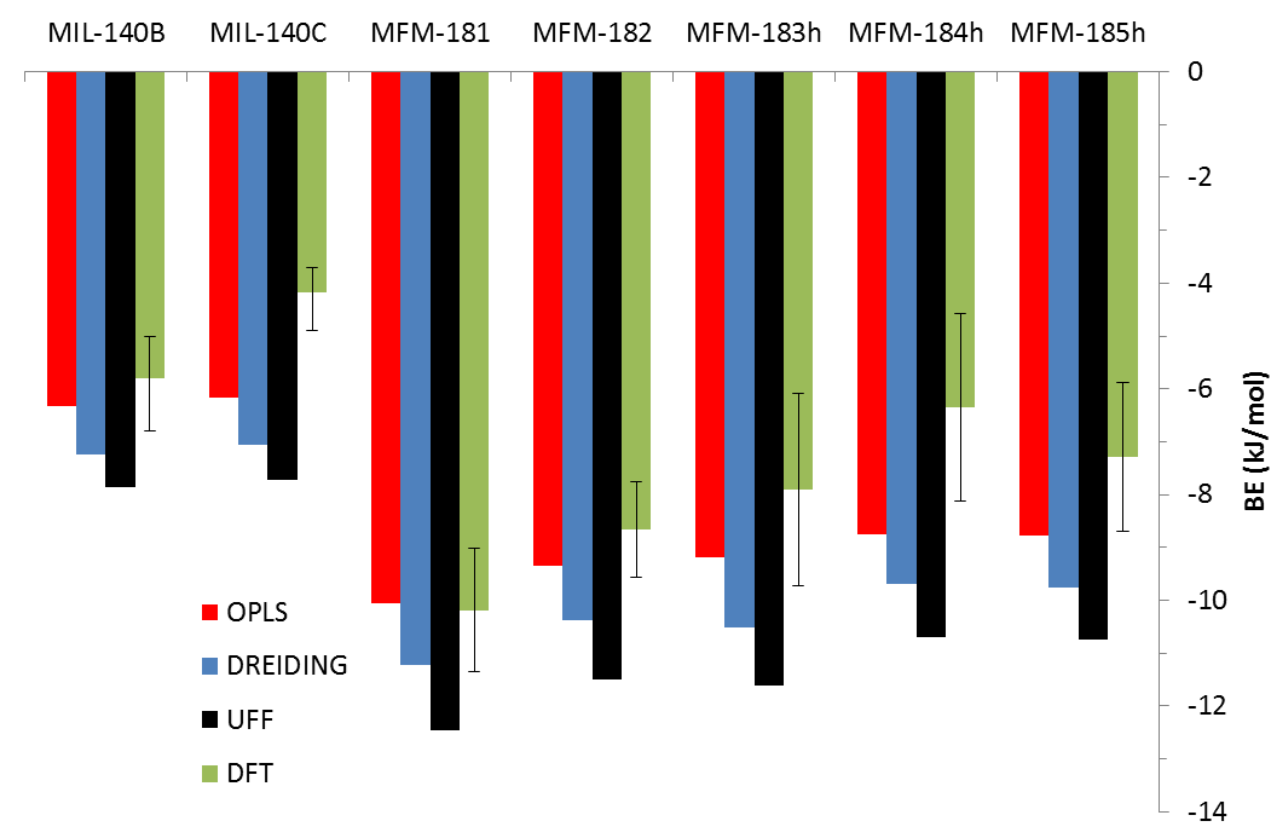

Figure $5-\mathrm{CH}_{4}$-Ligand binding energies for carbon-rich ligands as predicted via DFT (green), DREIDING (blue), OPLS (red) and UFF (black). Bars indicate the upper and lower limits of observed DFT binding sites.

In the case of carbon-rich ligands (Fig. 5), it is clear that while all three generic force fields are in the right ball-park, they tend to return a slightly stronger binding energy (BE) than that predicted by DFT. The smallest discrepancy is observed for OPLS, over-predicting by $1.2 \mathrm{~kJ} / \mathrm{mol}(\sim 20 \%)$ on 
average compared to the average DFT BE. It should be noted, however, that with the exception of the NDC ligand, OPLS predictions all fall within the range of BEs returned from DFT. Similarly, while DREIDING over-predicts the binding energy by an average of $2.2 \mathrm{~kJ} / \mathrm{mol}$ ( 35\%) compared to the DFT average, it is less than $1 \mathrm{~kJ} / \mathrm{mol}$ out when compared to the strongest binding sites predicted from DFT. It is clear, however, that the interaction strengths predicted from UFF are large, even when compared to the strongest sites observed in DFT (an over-prediction of $\sim 25 \%$ compared to the strongest DFT sites and $\sim 48 \%$ when compared to the DFT average).

In addition to evaluating the binding energy predicted by the three force fields, the predicted binding locations and spatial dependence of interaction energy were compared to those determined from DFT calculations. It should be noted that as all three force fields share a common mathematical form (the 12-6 Lennard-Jones potential), their predicted potential energy surfaces are very similar in shape. In fact, the variation in predicted binding location for each FF is less than the $0.2 \AA$ accuracy of the calculations employed in this study. As illustrated for the NDC ligand (Fig. 6), excellent agreement was observed between DFT and FF in terms of position with respect to the aromatic core of the ligand, although all three FFs consistently predicted a shorter $\mathrm{CH}_{4}$-ligand separation distance than in DFT (a discrepancy of 0.4 to $0.6 \AA$ on average). This was found to be the case for all of the carbon-rich ligands included in this study and suggests that, in principal, most generic 12-6 $\mathrm{LJ}$ potentials should be capable of predicting binding location for these types of systems. While UFF, DREIDING and OPLS are thus equally capable of predicting chemically meaningful binding locations for methane near organic ligands which are primarily carbocyclic in nature, OPLS and DREIDING would appear to offer the most accurate description of the interaction strength.

(a)
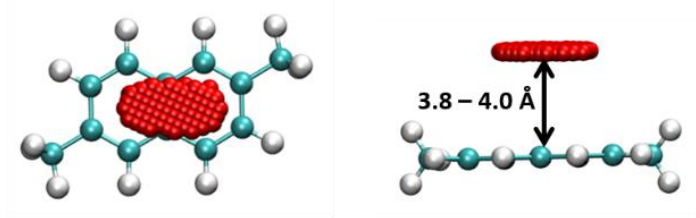

(b)
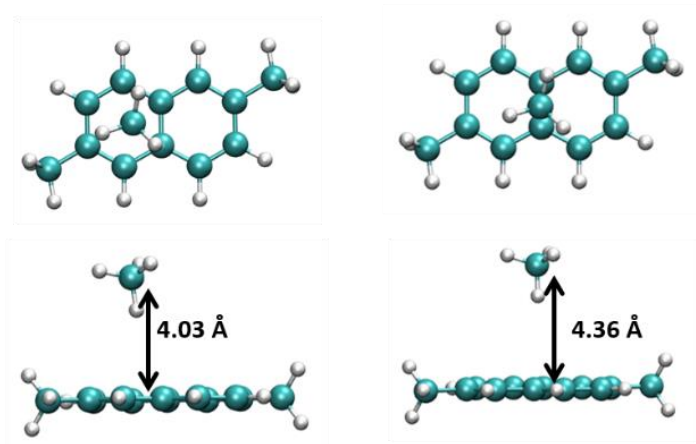

Figure 6 - Strongest $\mathrm{CH}_{4}$-NDC interaction sites as predicted by (a) $\mathrm{LJ}$ force fields and (b) DFT.

In the case of ligands with significant heterogeneity in their composition, such as those which incorporate $\mathrm{S}-, \mathrm{N}$ - or O-heteroatoms or functional groups (such as the azo-, amide and amine moieties in this study), the combination of the standard MOF force fields with TraPPE-UA $\mathrm{CH}_{4}$ generally performs well in predicting the interaction strength and location of the strongest binding sites observed in DFT (Fig. 7). As observed for the carbon-rich fragments, using UFF leads to a significant over-prediction of binding strength (by 5-45\%, and $17 \%$ on average). In contrast to the carbon-rich fragments, however, DREIDING and OPLS are seen to perform almost identically in cases where 
nitrogen or oxygen atoms are present in the fragment. Both force fields over-predict by 7 to $8 \%$ on average when compared to DFT, but, with the exception of the heterocyclic ligands of MFM-183 and MFM-185, fall within $1.2 \mathrm{~kJ} / \mathrm{mol}$ of the DFT predictions.

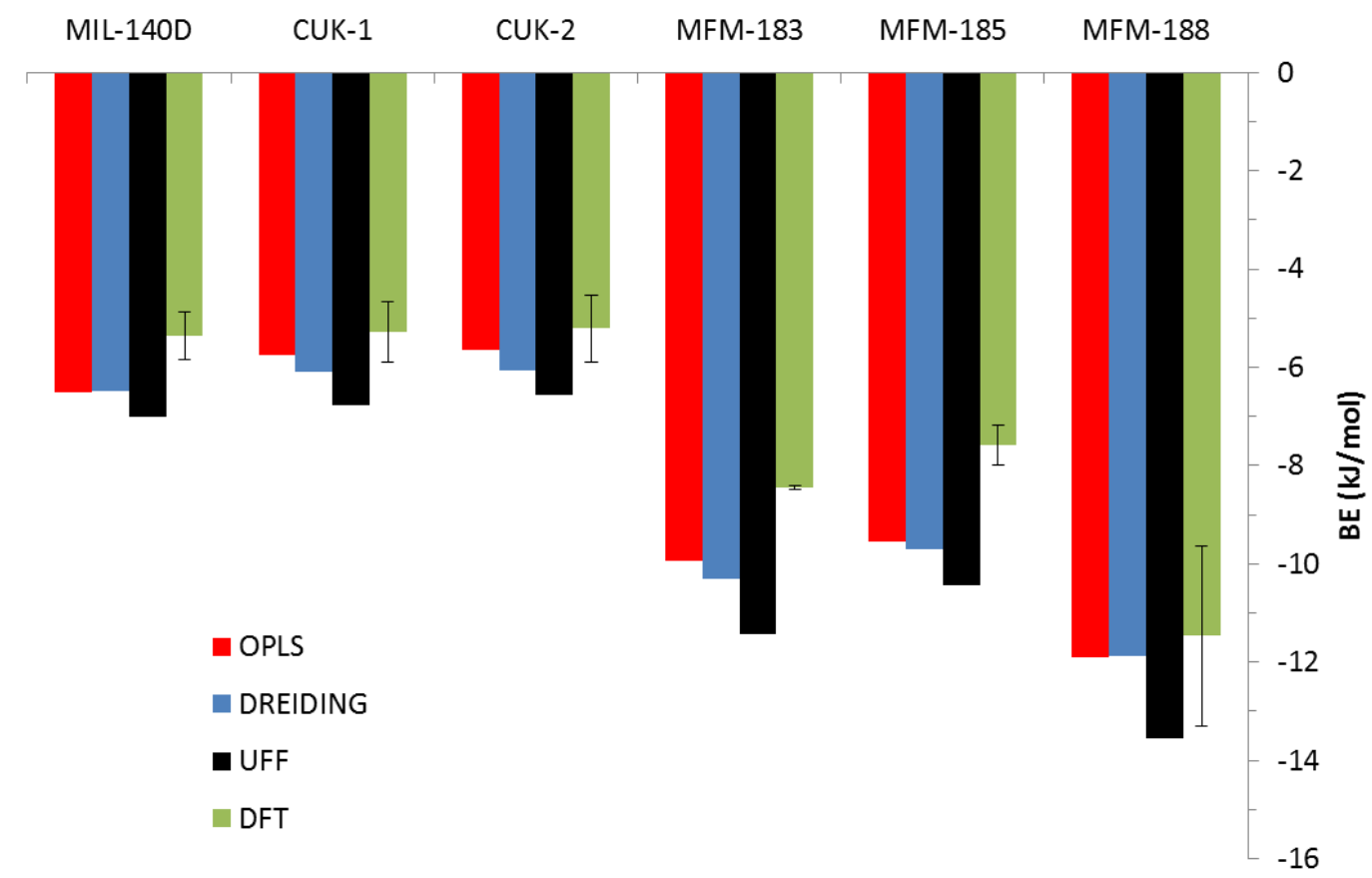

Figure $7-\mathrm{CH}_{4}$-Ligand binding energies for $\mathrm{N}$-, $\mathrm{O}$ and $\mathrm{S}$-containing ligands as predicted by DFT (green), DREIDING (blue), OPLS (red) and UFF (black). Bars indicate the upper and lower limits of observed DFT binding sites.

The cases of CUK-1/2 and MFM-183/5 are particularly interesting. These fragments contain heterocycles with either one (pyridine; CUK1/2) or two (pyrazine; MFM-183/5) N-heteroatoms per ring. In each case, the strongest binding location for $\mathrm{CH}_{4}$ was found to be directly above the heterocycle in both DFT and FF-based calculations. The magnitude of over-prediction in interaction strength from FF-based calculations varies significantly, however. For the low nitrogen content CUK1/2 fragments, both DREIDING and OPLS predict the interaction strength extremely well, both falling within the range of BEs observed in DFT. In the case of pyrazine-containing fragments, however, both force fields over-predict considerably (by 17-28\%). The strength of interaction between methane and $\mathrm{N}$ - or O-containing heterocyclic ligands is thus heavily influenced by the electronic structure of the heterocycle and care should be taken when relying on classical simulations to extract quantitative energetic information in MOFs with complex heterocyclic ligands.

In our previous work, methane binding around the heterocyclic ligands of the MFM-18X series was shown to be heavily influenced by weak hydrogen bonding between methane and the $\mathrm{N}$ - or $\mathrm{O}$ heteroatoms[33]. It should be remembered that a simple LJ potential should not be expected to be able to predict these types of interactions. In the case of the ligands of MFM-183/5, all three force fields were actually found to capture the strength of interaction and $\mathrm{CH}_{4}$-ligand separation surprisingly well in the regions where weak N--H hydrogen bonding was observed in DFT (Fig. 8) but over-predicted the strength of interaction near the O-heteroatom by $\sim 1.5 \mathrm{~kJ} / \mathrm{mol}(34 \%)$. Employing a more complex model to describe methane (OPLS-AA, which includes both $\mathrm{L}$ and Coulombic potential terms) did not 
result in an improvement. All binding sites - both above the aromatic core and in regions in which weak hydrogen bonding is to be expected - are now over-predicted by $25-50 \%$ (an increase from the 17 to $35 \%$ over-prediction observed for FF/TraPPE-UA).

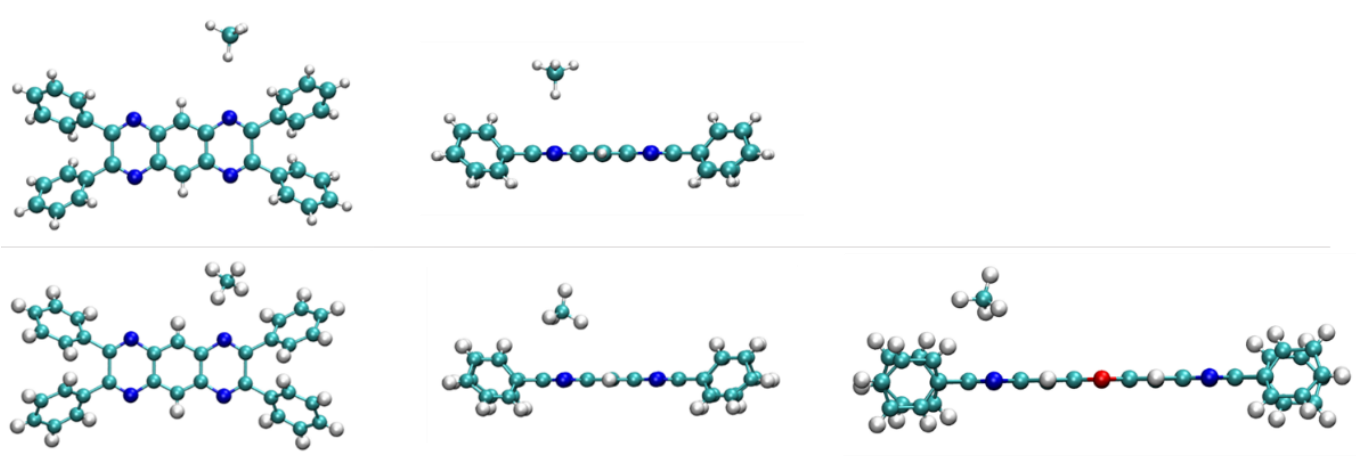

Figure 8 - Binding of $\mathrm{CH}_{4}$ with the MFM-183 and MFM-185 fragments predicted via DFT (top) and FF/OPLS-AA (bottom). Note that binding above the pyrazine ring of MFM-185 (bottom-right image) could not be reproduced in DFT calculations.

Interestingly, the preferred $\mathrm{CH}_{4}$ orientations predicted by FF/OPLS-AA simulations do not match those observed in DFT (Fig. 8). The interaction between the partial positive charge on the hydrogen of $\mathrm{CH}_{4}$ and either the $\pi$-electrons of the aromatic or partial negative charge of the heteroatom leads to $\mathrm{CH}_{4}$ aligning itself such that a single $\mathrm{H}$ atom is directed towards the ligand. In the case of OPLS-AA $\mathrm{CH}_{4}$, methane tended to align itself with 2-3 hydrogen atoms pointed towards the ligand in order to maximise the $\mathrm{L}$ component of the force field - i.e. the opposite of that predicted by DFT. Not only does the inclusion of point charges on methane fail to lead to an improvement in accuracy in the description of ligand-guest interaction energy, it also predicts methane orientations which are inconsistent with DFT-based predictions. Furthermore, both UA and OPLS-AA treatments of $\mathrm{CH}_{4}$ predict strong binding of methane directly above the pyrazine ring of the MFM-185 fragment - a site which could not be replicated in DFT calculations[33]. Therefore, for systems in which complex, non- $L$ type interactions may be present, quantum chemical calculations represent an excellent, complementary tool for investigation and validation of classical predictions.

Although OPLS was seen to consistently over-predict $\mathrm{CH}_{4}$ adsorption isotherms at low loading compared to both DREIDING and to experimental data (Figs. 1 and 2 ), it was found to be the bestperforming FF in terms of predicting the interaction of methane with the organic core of the MOF. The major difference between the two cases is the presence of metal oxide clusters in the GCMC simulations - the fragments investigated via DFT excluded the carboxylate groups. Further $\mathrm{BE}$ calculations were thus undertaken for the $\mathrm{CH}_{4}$--Zn-benzoate cluster, representative of the metalligand combination of IRMOF-1 (Fig. 8). As was the case for the organic fragments, all three force fields are able to correctly reproduce the binding locations observed in DFT - sites which have been previously explored by Dubbeldam et al [55]. Furthermore, all three force fields performed reasonably well in reproducing the binding energies predicted by DFT, with DREIDING correctly predicting the DFT binding energies to within $1 \mathrm{~kJ} / \mathrm{mol}$ across the system. Both UFF and OPLS over-predicted by $1-2 \mathrm{~kJ} / \mathrm{mol}$ in regions in which the primary interaction is with the benzene rings (Fig. 9a and 9c). Near the carboxylate groups (Fig. 8b), however, OPLS significantly over-predicted (2-3 kJ/mol; $20-30 \%$ ) in 
comparison to DFT and the other two force fields, primarily a result of the much higher $\varepsilon_{i j} / k_{B}$ parameter for the carboxylate oxygen in OPLS (105.7 K) compared to the other two force fields $(30.2$ $\mathrm{K}$ in UFF; $48.2 \mathrm{~K}$ in DREIDING).
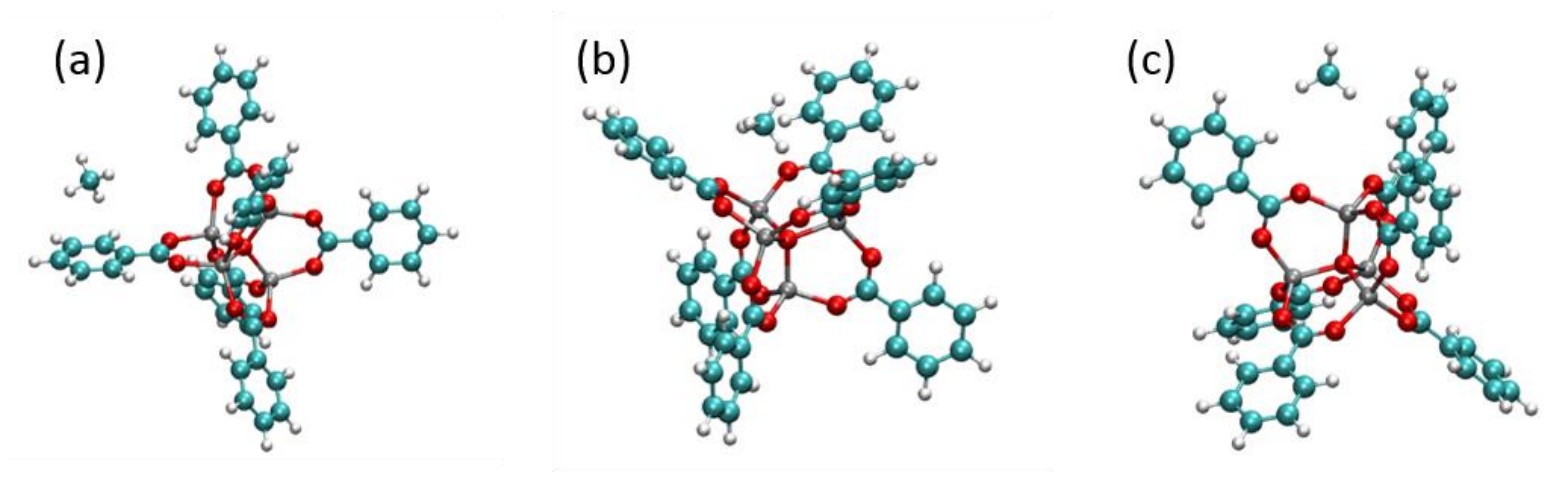

Figure 9 - Methane binding near the Zn-benzoate cluster, identified via DFT: (a) above a benzene ring; (b) sited in the 'corner' formed where three benzoate moieties intersect; (c) between two benzene rings, interacting primarily with the edges of the rings and oxygen atoms.

These preliminary results suggest that while the OPLS force field will produce an isotherm which will often look qualitatively correct, a significant over-estimation of the adsorption isotherm at low pressure is likely in any MOF using carboxylic acid as a coordinating group. Any subsequent analysis of preferred adsorption sites will be artificially skewed towards these regions of the MOF. This can be seen clearly in the work of Yang and Zhong[50], in which their re-parameterisations of the OPLS force field for CuBTC and IRMOF-1 primarily affected the $\varepsilon_{i i}$ parameter for the carboxylate oxygen.

Although UFF and OPLS perform very similarly in the prediction of isotherms - both overestimate adsorption at low loading by $\sim 30-50 \%$ in the cases studied - they do not appear to overestimate for the same reasons. While OPLS appears to capture interactions near the ligand well, it significantly over-estimates the interaction near carboxylate groups. Comparison of FF and DFT binding energies suggests that UFF over-estimates the interaction of $\mathrm{CH}_{4}$ with all regions of the framework by a similar magnitude. UFF may, therefore, be expected to still give qualitatively accurate predictions of the relative importance of different adsorption locations within the structure for most systems. It has been shown, however, that the reliability of classical force fields in predictions of methane binding near the metal cluster of a MOF is strongly dependent on the metal and its coordination state $[15,56,57,58]$, particularly for systems in which open-metal sites are present, and care should be taken in the interpretation of simulation data in these cases.

\section{Conclusions}

In this multi-level computational study, the suitability of generic force fields for use in the prediction of methane adsorption isotherms and adsorption mechanisms in MOFs at low coverage has been evaluated through a combination of classical and quantum chemical simulations. We demonstrate that while all three commonly used generic force fields tested in this work (DREIDING, UFF and OPLS-AA) are suitable for the qualitative prediction of adsorption isotherms, DREIDING 
provides superior quantitative agreement with experimental data, confirming the general literature consensus. We also, show, however, that DREIDING over-estimates the adsorbed amount by up to $25 \%$ on average for fractional loadings less than 0.5 . Furthermore, we demonstrate that selecting a cut-off radius of less than $17 \AA(4.5 \sigma)$ is likely to introduce a systematic and statistically significant underestimation in the amount adsorbed when compared to the converged result. This underestimation is relatively minor (5-10\%) and will further depend upon the implementation of the cut-off. In the present work, the $\mathrm{LJ}$ term was simply truncated at $r_{c}$. Alternatively, one may shift the potential to produce a smooth decay to zero at $r_{c}$ and/or choose to include a further tail correction to the $L$ energy.

Comparison of DFT and FF-based simulations of gas-ligand binding has shown that DREIDING and, in particular, OPLS-AA are capable of predicting the binding location and binding energy to a high degree of accuracy (to within $0.5 \AA$ and $1-2 \mathrm{~kJ} / \mathrm{mol}$ ). The level of accuracy attained by FF predictions decreases significantly in the case of ligands containing high concentrations of nitrogen or oxygen, however, and we recommend treating quantitative FF predictions of gas binding in these types of MOF systems with a certain degree of scepticism.

Based both on the results presented in the present work and the literature data summarised herein, we are able to make several suggestions as to best practice in choice of generic force field for predictions of methane adsorption in MOFs:

- UFF is likely to over-estimate both the adsorption isotherm and the interaction of methane with organic ligands by a significant amount and we would advise against relying on this force field for quantitative predictions of low-coverage adsorption properties. It is possible that the scaling factors suggested by Fairen-Jimenez[51] and Pérez-Peritello[23] may satisfactorily address this shortcoming, although this was not explored in the present work.

- While OPLS-AA performed well in predictions of gas-ligand binding, it is likely to over-estimate gas adsorption in MOFs which use carboxylic acid as a coordinating group and did not offer a significant improvement over UFF or DREIDING in the prediction of adsorption isotherms.

- DREIDING offered the best performance of the three tested force fields in the prediction of adsorption isotherms in all the systems considered in this study, and in literature studies in which more than one force field was evaluated[23, 48, 57]. It also performed very well in the prediction of gas-ligand interactions and, as it considers each element to have only one set of $\mathrm{L}$ parameters, has the advantage of being easily implemented in simulations.

Given the huge number of potential MOF structures, the present work is not a comprehensive study of methane adsorption in MOFs but intended to guide researchers in the selection of appropriate force fields for adsorption simulations and highlight some of the limitations and potential pit-falls of these approaches. Computational tools are - rightly - becoming more commonplace in the search for high-performance MOF adsorbents but, as with all tools, need to be used appropriately.

\section{Supporting Information}

Chemical structure of molecular fragments studied; $\mathrm{CH}_{4}$ and MOF $\mathrm{L}$ parameters; simulated and experimental adsorption isotherms.

\section{Acknowledgements}


EB gratefully acknowledges funding from ERC (Consolidator grant 307755 FIN) and EPSRC. The authors acknowledge support from the University of Nottingham's high-performance computing facilities. This research made use of the Balena High Performance Computing Service at the University of Bath. The authors are grateful to Prof. T. Düren for fruitful discussions on the choice of cut-off radius in $\mathrm{L}$ interactions.

\section{References}

1. Barea E, Montoro C, Navarro JAR. Toxic gas removal - metal-organic frameworks for the capture and degradation of toxic gases and vapours. Chem Soc Rev. 2014;43:5419-30.

2. Allendorf MD, Stavila V. Crystal engineering, structure-function relationships, and the future of metal-organic frameworks. Crystengcomm. 2015;17:229-46.

3. Farrusseng D. Metal-Organic Frameworks: Applications from Catalysis to Gas Storage. Weinheim, Germany: Wiley; 2011.

4. Lee J, Farha OK, Roberts J, Scheidt KA, Nguyen ST, Hupp JT. Metal-organic framework materials as catalysts. Chem Soc Rev. 2009;38:1450-9.

5. Furukawa H, Cordova KE, O'Keeffe M, Yaghi OM. The Chemistry and Applications of MetalOrganic Frameworks. Science. 2013;341:974.

6. Li JR, Kuppler RJ, Zhou HC. Selective gas adsorption and separation in metal-organic frameworks. Chem Soc Rev. 2009;38:1477-504.

7. Colon YJ, Snurr RQ. High-throughput computational screening of metal-organic frameworks. Chem Soc Rev. 2014;43:5735-49.

8. Coudert FX, Fuchs AH. Computational characterization and prediction of metal-organic framework properties. Coord Chem Rev. 2016;307:211-36.

9. Duren T, Bae YS, Snurr RQ. Using molecular simulation to characterise metal-organic frameworks for adsorption applications. Chem Soc Rev. 2009;38:1237-47.

10. Getman RB, Bae Y-S, Wilmer CE, Snurr RQ. Review and Analysis of Molecular Simulations of Methane, Hydrogen, and Acetylene Storage in Metal-Organic Frameworks. Chem Rev. 2012;112:703-23.

11. First EL, Floudas CA. MOFomics: Computational pore characterization of metal-organic frameworks. Microporous Mesoporous Mater. 2013;165:32-9.

12. Düren T, Sarkisov L, Yaghi OM, Snurr RQ. Design of New Materials for Methane Storage. Langmuir. 2004;20:2683-9.

13. Sarkisov L, Duren T, Snurr RQ. Molecular modelling of adsorption in novel nanoporous metal-organic materials. Mol Phys. 2004;102:211-21.

14. Bichoutskaia E, Suyetin M, Bound M, Yan Y, Schroeder M. Methane Adsorption in MetalOrganic Frameworks Containing Nanographene Linkers: A Computational Study. J Phys Chem C. 2014;118:15573-80.

15. Chen LJ, Grajciar L, Nachtigall P, Duren T. Accurate Prediction of Methane Adsorption in a Metal-Organic Framework with Unsaturated Metal Sites by Direct Implementation of an ab Initio Derived Potential Energy Surface in GCMC Simulation. J Phys Chem C. 2011;115:23074-80. 
16. Cossi M, Gatti G, Canti L, Tei L, Errahali M, Marchese L. Theoretical Prediction of High Pressure Methane Adsorption in Porous Aromatic Frameworks (PAFs). Langmuir. 2012;28:14405-14.

17. Fernandez M, Woo TK, Wilmer CE, Snurr RQ. Large-Scale Quantitative Structure-Property Relationship (QSPR) Analysis of Methane Storage in Metal-Organic Frameworks. J Phys Chem C. 2013;117:7681-9.

18. Gomez-Gualdron DA, Gutov OV, Krungleviciute V, Borah B, Mondloch JE, Hupp JT, Yildirim T, Farha OK, Snurr RQ. Computational Design of Metal-Organic Frameworks Based on Stable Zirconium Building Units for Storage and Delivery of Methane. Chem Mater. 2014;26:5632-9.

19. Wilmer CE, Leaf M, Lee CY, Farha OK, Hauser BG, Hupp JT, Snurr RQ. Large-scale screening of hypothetical metal-organic frameworks. Nat Chem. 2012;4:83-9.

20. Garberoglio G. Computer Simulation of the Adsorption of Light Gases in Covalent Organic Frameworks. Langmuir. 2007;23:12154-8.

21. Garberoglio G, Skoulidas Al, Johnson JK. Adsorption of Gases in Metal Organic Materials: Comparison of Simulations and Experiments. J Phys Chemi B. 2005;109:13094-103.

22. Yang QY, Wiersum AD, Jobic H, Guillerm V, Serre C, Llewellyn PL, Maurin G. Understanding the Thermodynamic and Kinetic Behavior of the $\mathrm{CO} 2 / \mathrm{CH} 4$ Gas Mixture within the Porous Zirconium Terephthalate UiO-66(Zr): A Joint Experimental and Modeling Approach. J Phys Chem C. 2011;115:13768-74.

23. Pérez-Pellitero J, Amrouche H, Siperstein FR, Pirngruber G, Nieto-Draghi C, Chaplais G, Simon-Masseron A, Bazer-Bachi D, Peralta D, Bats N. Adsorption of $\mathrm{CO} 2, \mathrm{CH} 4$, and N2 on Zeolitic Imidazolate Frameworks: Experiments and Simulations. Chemistry - A European Journal. 2010;16:1560-71.

24. Rappe AK, Casewit CJ, Colwell KS, Goddard WA, Skiff WM. Uff, a Full Periodic-Table ForceField for Molecular Mechanics and Molecular-Dynamics Simulations. J Am Chem Soc. 1992;114:10024-35.

25. Mayo SL, Olafson BD, Goddard WA. Dreiding - a Generic Force-Field for Molecular Simulations. J Phys Chem. 1990;94:8897-909.

26. Jorgensen WL, Laird ER, Nguyen TB, Tiradorives J. MONTE-CARLO SIMULATIONS OF PURE LIQUID SUBSTITUTED BENZENES WITH OPLS POTENTIAL FUNCTIONS. J Comput Chem. 1993;14:20615.

27. Jorgensen WL, Maxwell DS, Tirado-Rives J. Development and Testing of the OPLS All-Atom Force Field on Conformational Energetics and Properties of Organic Liquids. J Am Chem Soc. 1996;118:11225-36.

28. Yang Q, Liu D, Zhong C, Li J-R. Development of Computational Methodologies for MetalOrganic Frameworks and Their Application in Gas Separations. Chem Rev. 2013.

29. Goerigk L, Grimme S. A thorough benchmark of density functional methods for general main group thermochemistry, kinetics, and noncovalent interactions. PCCP. 2011;13:6670-88.

30. Shao YH, Gan ZT, Epifanovsky E, Gilbert ATB, Wormit M, Kussmann J, Lange AW, Behn A, Deng J, Feng XT, Ghosh D, Goldey M, Horn PR, Jacobson LD, Kaliman I, Khaliullin RZ, Kus T, Landau A, Liu J, Proynov El, Rhee YM, Richard RM, Rohrdanz MA, Steele RP, Sundstrom EJ, Woodcock HL, Zimmerman PM, Zuev D, Albrecht B, Alguire E, Austin B, Beran GJO, Bernard YA, Berquist E, Brandhorst K, Bravaya KB, Brown ST, Casanova D, Chang CM, Chen YQ, Chien SH, Closser KD, Crittenden DL, Diedenhofen M, DiStasio RA, Do H, Dutoi AD, Edgar RG, Fatehi S, Fusti-Molnar L, 
Ghysels A, Golubeva-Zadorozhnaya A, Gomes J, Hanson-Heine MWD, Harbach PHP, Hauser AW, Hohenstein EG, Holden ZC, Jagau TC, Ji HJ, Kaduk B, Khistyaev K, Kim J, Kim J, King RA, Klunzinger P, Kosenkov D, Kowalczyk T, Krauter CM, Lao KU, Laurent AD, Lawler KV, Levchenko SV, Lin CY, Liu F, Livshits E, Lochan RC, Luenser A, Manohar P, Manzer SF, Mao SP, Mardirossian N, Marenich AV, Maurer SA, Mayhall NJ, Neuscamman E, Oana CM, Olivares-Amaya R, O'Neill DP, Parkhill JA, Perrine TM, Peverati R, Prociuk A, Rehn DR, Rosta E, Russ NJ, Sharada SM, Sharma S, Small DW, Sodt A, Stein $T$, Stuck D, Su YC, Thom AJW, Tsuchimochi T, Vanovschi V, Vogt L, Vydrov O, Wang T, Watson MA, Wenzel J, White A, Williams CF, Yang J, Yeganeh S, Yost SR, You ZQ, Zhang IY, Zhang X, Zhao Y, Brooks BR, Chan GKL, Chipman DM, Cramer CJ, Goddard WA, Gordon MS, Hehre WJ, Klamt A, Schaefer HF, Schmidt MW, Sherrill CD, Truhlar DG, Warshel A, Xu X, Aspuru-Guzik A, Baer R, Bell AT, Besley NA, Chai JD, Dreuw A, Dunietz BD, Furlani TR, Gwaltney SR, Hsu CP, Jung YS, Kong J, Lambrecht DS, Liang WZ, Ochsenfeld C, Rassolov VA, Slipchenko LV, Subotnik JE, Van Voorhis T, Herbert JM, Krylov Al, Gill PMW, Head-Gordon M. Advances in molecular quantum chemistry contained in the Q-Chem 4 program package. Mol Phys. 2015;113:184-215.

31. Becke AD. DENSITY-FUNCTIONAL THERMOCHEMISTRY .3. THE ROLE OF EXACT EXCHANGE. J Chem Phys. 1993;98:5648-52.

32. Vogiatzis KD, Klopper W, Friedrich J. Non-covalent Interactions of $\mathrm{CO} 2$ with Functional Groups of Metal-Organic Frameworks from a CCSD(T) Scheme Applicable to Large Systems. J Chem Theory Comput. 2015;11:1574-84.

33. Henley A, Lennox MJ, Easun TL, Moreau F, Schröder M, Besley E. Computational Evaluation of the Impact of Incorporated Nitrogen and Oxygen Heteroatoms on the Affinity of Polyaromatic Ligands for Carbon Dioxide and Methane in Metal-Organic Frameworks. J Phys Chem C. 2016;120:27342-48.

34. Grajciar L, Nachtigall P, Bludský O, Rubeš M. Accurate Ab Initio Description of Adsorption on Coordinatively Unsaturated Cu2+ and Fe3+ Sites in MOFs. J Chem Theory Comput. 2015;11:230-8.

35. Supronowicz B, Mavrandonakis A, Heine T. Interaction of Small Gases with the Unsaturated Metal Centers of the HKUST-1 Metal Organic Framework. J Phys Chem C. 2013;117:14570-8.

36. Boys SF, Bernardi F. CALCULATION OF SMALL MOLECULAR INTERACTIONS BY DIFFERENCES OF SEPARATE TOTAL ENERGIES - SOME PROCEDURES WITH REDUCED ERRORS. Mol Phys. 1970;19:553-\&.

37. Martin MG, Siepmann JI. Transferable potentials for phase equilibria. 1. United-atom description of n-alkanes. J Phys Chem B. 1998;102:2569-77.

38. Amirjalayer S, Schmid R. Adsorption of Hydrocarbons in Metal-Organic Frameworks: A Force Field Benchmark on the Example of Benzene in Metal-Organic Framework 5. J Phys Chem C. 2012;116:15369-77.

39. Haldoupis E, Borycz J, Shi HL, Vogiatzis KD, Bai P, Queen WL, Gagliardi L, Siepmann JI. Ab Initio Derived Force Fields for Predicting $\mathrm{CO} 2$ Adsorption and Accessibility of Metal Sites in the Metal-Organic Frameworks M-MOF-74 (M = Mn, Co, Ni, Cu). J Phys Chem C. 2015;119:16058-71.

40. Kulkarni AR, Sholl DS. DFT-Derived Force Fields for Modeling Hydrocarbon Adsorption in MIL-47(V). Langmuir. 2015;31:8453-68.

41. Casewit CJ, Colwell KS, Rappe AK. APPLICATION OF A UNIVERSAL FORCE-FIELD TO ORGANICMOLECULES. J Am Chem Soc. 1992;114:10035-46.

42. Rappe AK, Colwell KS, Casewit CJ. APPLICATION OF A UNIVERSAL FORCE-FIELD TO METALCOMPLEXES. Inorg Chem. 1993;32:3438-50. 
43. Potoff JJ, Errington JR, Panagiotopoulos AZ. Molecular simulation of phase equilibria for mixtures of polar and non-polar components. Mol Phys. 1999;97:1073-83.

44. Ozturk TN, Keskin S. Computational Screening of Porous Coordination Networks for Adsorption and Membrane-Based Gas Separations. J Phys Chem C. 2014;118:13988-97.

45. Wang S. Comparative Molecular Simulation Study of Methane Adsorption in Metal-Organic Frameworks. Energy \& Fuels. 2007;21:953-6.

46. Sarkisov L. Toward Rational Design of Metal-Organic Frameworks for Sensing Applications: Efficient Calculation of Adsorption Characteristics in Zero Loading Regime. J Phys Chem C. 2012;116:3025-33.

47. Gupta A, Chempath S, Sanborn MJ, Clark LA, Snurr RQ. Object-oriented programming paradigms for molecular modeling. Molecular Simulation. 2003;29:29-46.

48. Pillai RS, Pinto ML, Pires J, Jorge M, Gomes JRB. Understanding Gas Adsorption Selectivity in IRMOF-8 Using Molecular Simulation. ACS Applied Materials \& Interfaces. 2015;7:624-37.

49. Moreau F, Kolokolov DI, Stepanov AG, Easun TL, Dailly A, Lewis W, Blake AJ, Nowell H, Lennox MJ, Besley E, Yang S, Schroder M. Engineering Porosity and Rotational Dynamics in a Series of Octacarboxylate Metal-Organic Frameworks. PNAS (Accepted). 2017.

50. Yang QY, Zhong CL. Molecular simulation of carbon dioxide/methane/hydrogen mixture adsorption in metal-organic frameworks. J Phys Chem B. 2006;110:17776-83.

51. Fairen-Jimenez D, Galvelis R, Torrisi A, Gellan AD, Wharmby MT, Wright PA, Mellot-Draznieks $C$, Duren T. Flexibility and swing effect on the adsorption of energy-related gases on ZIF-8: combined experimental and simulation study. Dalton Transactions. 2012;41:10752-62.

52. Allen P, Tildesley DJ. Computer simulation of liquids. Oxford, UK: Oxford University Press; 1987.

53. Trokhymchuk A, Alejandre J. Computer simulations of liquid/vapor interface in LennardJones fluids: Some questions and answers. The Journal of Chemical Physics. 1999;111:8510-23.

54. Pàmies JC, McCabe C, Cummings PT, Vega LF. Coexistence Densities of Methane and Propane by Canonical Molecular Dynamics and Gibbs Ensemble Monte Carlo Simulations. Molecular Simulation. 2003;29:463-70.

55. Dubbeldam D, Frost $\mathrm{H}$, Walton KS, Snurr RQ. Molecular simulation of adsorption sites of light gases in the metal-organic framework IRMOF-1. Fluid Phase Equilib. 2007;261:152-61.

56. Fischer M, Gomes JRB, Jorge M. Computational approaches to study adsorption in MOFs with unsaturated metal sites. Molecular Simulation. 2014;40:537-56.

57. Rana MK, Koh HS, Zuberi H, Siegel DJ. Methane Storage in Metal-Substituted Metal-Organic Frameworks: Thermodynamics, Usable Capacity, and the Impact of Enhanced Binding Sites. J Phys Chem C. 2014;118:2929-42.

58. Koh HS, Rana MK, Wong-Foy AG, Siegel DJ. Predicting Methane Storage in Open-Metal-Site Metal-Organic Frameworks. J Phys Chem C. 2015;119:13451-8. 